\title{
Congruences and group congruences on a semigroup
}

\author{
Roman S. Gigoń
}

Received: 13 September 2011 / Accepted: 1 August 2012 / Published online: 15 August 2012

(C) The Author(s) 2012. This article is published with open access at Springerlink.com

\begin{abstract}
We show that there is an inclusion-preserving bijection between the set of all normal subsemigroups of a semigroup $S$ and the set of all group congruences on $S$. We describe also group congruences on $E$-inversive $(E$-)semigroups. In particular, we generalize the result of Meakin (J. Aust. Math. Soc. 13:259-266, 1972) concerning the description of the least group congruence on an orthodox semigroup, the result of Howie (Proc. Edinb. Math. Soc. 14:71-79, 1964) concerning the description of $\rho \vee \sigma$ in an inverse semigroup $S$, where $\rho$ is a congruence and $\sigma$ is the least group congruence on $S$, some results of Jones (Semigroup Forum 30:1-16, 1984) and some results contained in the book of Petrich (Inverse Semigroups, 1984). Also, one of the main aims of this paper is to study of group congruences on $E$-unitary semigroups. In particular, we prove that in any $E$-inversive semigroup, $\mathcal{H} \cap \sigma \subseteq \kappa$, where $\kappa$ is the least $E$-unitary congruence. This result is equivalent to the statement that in an arbitrary $E$-unitary $E$-inversive semigroup $S, \mathcal{H} \cap \sigma=1_{S}$.
\end{abstract}

Keywords Group congruence $\cdot E$-inversive semigroup $\cdot E$-semigroup Idempotent-surjective semigroup · Eventually regular semigroup · Idempotent pure congruence $\cdot$ Idempotent-separating congruence $\cdot E$-unitary congruence

\section{Introduction and preliminaries}

Let $S$ be an inverse semigroup with semilattice of idempotents $E$. Define an inverse subsemigroup $N$ of $S$ to be normal if it is full (i.e., $E \subseteq N$ ), closed (i.e., $N \omega=N$, where $\omega: 2^{S} \rightarrow 2^{S}$ is a closure operator given by $A \omega=\{s \in S: \exists a \in A[$ as $\in A]\}$

Communicated by Marcel Jackson.

R.S. Gigoń (凶)

Institute of Mathematics and Computer Science, Wroclaw University of Technology,

Wyb. Wyspianskiego 27, 50-370 Wroclaw, Poland

e-mail: romekgigon@tlen.pl 
for all $A \subseteq S$ ), and self-conjugate (i.e., $s^{-1} N s \subseteq N$ for every $s \in S$ ). It follows from [11] (see p. 181) that there exists an inclusion-preserving bijection between the set of normal subsemigroups of $S$ and the set of group congruences on $S$. In fact, the relation $\rho_{N}=\left\{(a, b) \in S \times S: a b^{-1} \in N\right\}$ is a group congruence on $S$ and $\operatorname{ker} \rho_{N}=N$. These results were generalized in [9] and [16]. It is easy to see that $(a, b) \in \rho_{N}$ if and only if $a x, b x \in N$ for some $x \in S$.

The main purpose of the next section is a description of group congruences on a semigroup $S$ in the terms of some special subsemigroups of $S$. Our description is simpler than that of Dubreil (see 10.2 [1]) and a little more general than the description of Gomes [6] (nota bene our proof is simpler). We apply this description to determine group congruences (in particular, the least group congruence) on some special classes of semigroups; namely: $E$-inversive $(E$-)semigroups (in particular, idempotent-surjective ( $E$-)semigroups), eventually regular semigroups.

We divide this paper into seven sections. In Sect. 2 we describe group congruences on an arbitrary semigroup $S$ in the terms of normal subsemigroups of $S$ (see below for the definition). In Sect. 3 we investigate group congruences on $E$-inversive semigroups. In particular, we show that the least group congruence on an $E$-inversive semigroup exists (in general, this is false: see Example 1.2). In Sect. 4 and 5 we study group congruences on $E$-inversive $E$-semigroups and $E$-unitary $E$-inversive semigroups, respectively. Further, in Sect. 6 we use the results of Sect. 2 for an easy description of all group congruences on eventually regular semigroups (in terms of full, closed and self-conjugate subsemigroups) and we give some remarks on group congruences on inverse semigroups. Finally, in Sect. 7, some remarks on the hypercore of a semigroup are given (see [8]).

Let $S$ be a semigroup. Denote by $\operatorname{Reg}(S)$ the set of regular elements of $S$, that is, $\operatorname{Reg}(S)=\{a \in S: a \in a S a\}$ and by $V(a)$ the set of inverses of $a \in S$, i.e., the set $\{x \in S: a=a x a, x=x a x\}$. Note that if $a \in S$ is regular, say $a=a x a$ for some $x \in S$, then $x a x \in V(a)$. Also, $S$ is called regular if $V(a) \neq \emptyset$ for every $a \in S$. Further, $S$ is said to be eventually regular if every element $a$ of $S$ has a regular power. In such a case, by $r(a)$ we shall mean the regular index of $a$, i.e., the least positive integer $n$ for which $a^{n} \in \operatorname{Reg}(S)$.

Let $S$ be a semigroup, $a \in S$. The set $W(a)=\{x \in S: x=x a x\}$ is called the set of all weak inverses of $a$ and so the elements of $W(a)$ will be called weak inverse elements of $a$. A semigroup $S$ is said to be $E$-inversive if for every $a \in S$ there exists $x \in S$ such that $a x \in E_{S}$, where $E_{S}$ (or briefly $E$ ) is the set of idempotents of $S$ (more generally, if $A \subseteq S$, then $E_{A}$ denotes the set of idempotents of $A$ ). It is easy to see that a semigroup $S$ is $E$-inversive if and only if $W(a)$ is nonempty for all $a \in S$. Hence if $S$ is $E$-inversive, then for every $a \in S$ there is $x \in S$ such that $a x, x a \in E_{S}$ (see $[19,20])$. Clearly, eventually regular semigroups are $E$-inversive. We remark that the class of eventually regular semigroups is very wide and contains the class of regular, group-bound (in particular, periodic, finite) semigroups. In [7] Hall observed that the set $\operatorname{Reg}(S)$ of a semigroup $S$ with $E_{S} \neq \varnothing$ forms a regular subsemigroup of $S$ if and only if the product of any two idempotents of $S$ is regular. In that case, $S$ is said to be an $R$-semigroup. Also, we say that $S$ is an E-semigroup if $E_{S}$ is a subsemigroup of $S$. Evidently, every $E$-semigroup is an $R$-semigroup. Finally, [eventually] regular $E$-semigroups are called [eventually] orthodox. 
A generalization of the concept of eventually regular will also prove convenient. Define a semigroup $S$ to be idempotent-surjective if whenever $\rho$ is a congruence on $S$ and $a \rho$ is an idempotent of $S / \rho$, then $a \rho$ contains some idempotent of $S$. It is well known that eventually regular semigroups are idempotent-surjective [2]. Further, we have the following known result [10] (we include a simple proof for completeness).

Result 1.1 Every idempotent-surjective semigroup $S$ is E-inversive.

Proof Let $a \in S$. From the definition of a Rees congruence on $S$ follows that the ideal $S a S$ has at least one idempotent, that is, $x a y=e \in E_{S}$, where $x, y \in S$. Hence exaye $=e$. Thus yex $=($ yex $) a($ yex $)$, so yex $\in W(a)$, as required.

A subset $A$ of $S$ is said to be (respectively) full; reflexive and dense if $E_{S} \subseteq A$; $\forall a, b \in S[a b \in A \Longrightarrow b a \in A]$ and $\forall s \in S \exists x, y \in S[s x, y s \in A]$. Also, define the closure operator $\omega$ on $S$ by $A \omega=\{s \in S: \exists a \in A[a s \in A]\}(A \subseteq S)$. We shall say that $A \subseteq S$ is closed (in $S$ ) if $A \omega=A$. Finally, a subsemigroup $N$ of a semigroup $S$ is normal if it is full, dense, reflexive and closed (if $N$ is normal, then we shall write $N \triangleleft S$ ). Moreover, if a subsemigroup of $S$ is full, dense and reflexive, then it is called seminormal [6].

By the kernel $\operatorname{ker} \rho$ of a congruence $\rho$ on a semigroup $S$ we shall mean the set $\left\{x \in S:\left(x, x^{2}\right) \in \rho\right\}$. Finally, denote by $\mathcal{C}(S)$ the complete lattice of all congruences on a semigroup $S$.

Example 1.2 Consider the semigroup of positive integers $(\mathbb{N},+$ ) (with respect to addition). It is well known that every group congruence on $\mathbb{N}$ is of the following form: $\rho_{n}=\{(k, l) \in \mathbb{N} \times \mathbb{N}: n \mid(k-l)\}(n>0)$. Note that $E_{\mathbb{N}}=\emptyset$, so a semigroup without idempotents possesses group congruences but $\mathbb{N}$ has not least group congruence. Also, $\operatorname{ker} \rho_{n}=n \rho_{n}=\{n, 2 n, 3 n, \ldots\}$.

\section{Group congruences-general case}

Let $S$ be a semigroup, $\rho \in \mathcal{C}(S)$. We say that $\rho$ is a group congruence if $S / \rho$ is a group. Denote by $\mathcal{G C}(S)$ the set of group congruences on $S$. Clearly, if $\rho \in \mathcal{G C}(S)$, then $\operatorname{ker} \rho$ is the identity of the group $S / \rho$. Finally, by $\mathcal{N}(S)$ we shall mean the set of all normal subsemigroups of $S$.

The following two lemmas are almost evident and we omit their easy proofs.

Lemma 2.1 Let $\rho$ be a group congruence on a semigroup $S$. Then $\operatorname{ker} \rho \triangleleft S$.

Lemma 2.2 Let $\rho_{1}, \rho_{2}$ be group congruences on a semigroup $S$. Then $\rho_{1} \subset \rho_{2}$ if and only if $\operatorname{ker} \rho_{1} \subset \operatorname{ker} \rho_{2}$.

Let $B$ be a nonempty subset of a semigroup $S$. Consider four relations on $S$ :

$$
\rho_{1, B}=\{(a, b) \in S \times S: \exists x \in S[a x, b x \in B]\} ;
$$




$$
\begin{aligned}
& \rho_{2, B}=\{(a, b) \in S \times S: \exists x, y \in B[a x=y b]\} ; \\
& \rho_{3, B}=\{(a, b) \in S \times S: \exists x \in S[x a, x b \in B]\} ; \\
& \rho_{4, B}=\{(a, b) \in S \times S: \exists x, y \in B[x a=b y]\} .
\end{aligned}
$$

Lemma 2.3 Let a subsemigroup $B$ of a semigroup $S$ be dense and reflexive. Then $\rho_{1, B}=\rho_{2, B}=\rho_{3, B}=\rho_{4, B}$.

Proof Let $(a, b) \in \rho_{2, B}$. Then $a x=y b$ for some $x, y \in B$. Also, $a s \in B$ for some $s \in S$, since $B$ is dense and so $s a \in B$, since $B$ is reflexive. Hence $a s y \in B$ and so $(s y) a \in B$. It follows that $(s y) b=s(y b)=s(a x)=(s a) x \in B$. Thus (sy) $a$, (sy) $b \in B$. We have just shown that $\rho_{2, B} \subset \rho_{3, B}$.

Conversely, if $x a, x b \in B$ for some $x \in S$, then $a x, b x \in B$ (since $B$ is reflexive), so $a(x b)=(a x) b$, where $a x, x b \in B$. Hence $(a, b) \in \rho_{2, B}$. Thus $\rho_{2, B}=\rho_{3, B}$.

Dually, $\rho_{1, B}=\rho_{4, B}$. Since $B$ is reflexive, then $\rho_{1, B}=\rho_{3, B}$.

If $B$ is a dense, reflexive subsemigroup of $S$, then we denote the above four relations by $\rho_{B}$. We have the following theorem.

Theorem 2.4 Let $B$ be a dense and reflexive subsemigroup of a semigroup $S$. Then the relation $\rho_{B}$ is a group congruence on $S$. Moreover, $B \subseteq B \omega=\operatorname{ker} \rho_{B}$. If $B$ is normal, then $B=\operatorname{ker} \rho_{B}$.

Conversely, if $\rho$ is a group congruence on $S$, then there exists a normal subsemigroup $N$ of $S$ such that $\rho=\rho_{N}$ (in fact, $\left.N=\operatorname{ker} \rho\right)$. Thus there exists an inclusionpreserving bijection between the set of all normal subsemigroups of $S$ and the set of all group congruences on $S$.

Proof Let $a \in S$. Since $B$ is dense, then there exists $x \in S$ such that $x a \in B$. Hence $\rho_{B}$ is reflexive. Obviously, $\rho_{B}$ is symmetric. Also, since $B$ is a semigroup, then $\rho_{B}$ is transitive. Consequently, $\rho_{B}$ is an equivalence relation on $S$. Moreover, $\rho_{B}$ is a left congruence on $S$. Indeed, let $(a, b) \in \rho_{B}, c \in S$. Then $a x, b x \in B$ and $z c \in B$ for some $x, z \in S$, so $z c a x, z c b x \in B$. It follows that $(c a)(x z),(c b)(x z) \in B$, since $B$ is reflexive. Therefore $(c a, c b) \in \rho_{B}$. By symmetry, $\rho_{B}$ is a right congruence on $S$. Finally, $S / \rho_{B}$ is a group. Indeed, let $a \in S, b \in B$ and $a x, x a \in B$ for some $x \in S$. Then bax $\in B$. Hence $x a, x(b a) \in B$, so $(b a, a) \in \rho_{B}$. Since $B$ is dense, then $S / \rho_{B}$ is a group, as required.

Since $b(b b)=(b b) b$ for every $b \in B$, then $B \subset \operatorname{ker} \rho_{B}$. Also, $B \omega=\operatorname{ker} \rho_{B}$. Indeed, let $s \in \operatorname{ker} \rho_{B}$. Then $(s, b) \in \rho_{B}$ for some $b \in B$. Hence $b_{1} s=b b_{2}$ for some $b_{1}, b_{2} \in B$. Thus $s \in B \omega$, so ker $\rho_{B} \subset B \omega$. Conversely, let $s \in B \omega$. Then $b s \in B$ for some $b \in B$. Since $b b \in B$, then $(s, b) \in \rho_{B}$, so $s \in \operatorname{ker} \rho_{B}$. Thus $B \omega \subset \operatorname{ker} \rho_{B}$, as exactly required. Finally, if $B$ is normal, then $B=B \omega$. Hence $B=\operatorname{ker} \rho_{B}$.

Conversely, let $\rho$ be a group congruence on $S$. By Lemma 2.1, $\operatorname{ker} \rho \triangleleft S$. Put $\operatorname{ker} \rho=N$. Then by Lemma 2.2, $\rho=\rho_{N}$, since $N=\operatorname{ker} \rho_{N}=\operatorname{ker} \rho$. It is now easy to see that the map $\phi: \mathcal{N}(S) \rightarrow \mathcal{G C}(S)$, where $N \phi=\rho_{N}$ for every $N \in \mathcal{N}(S)$, is an inclusion-preserving bijection between the set of all normal subsemigroups of $S$ and the set of all group congruences on $S$ (with the inverse $\phi^{-1}: \mathcal{G C}(S) \rightarrow \mathcal{N}(S)$, where 
$\rho \phi^{-1}=\operatorname{ker} \rho$ for all $\left.\rho \in \mathcal{G C}(S)\right)$. Note that $\phi^{-1}$ is an inclusion-preserving mapping, too.

Since the first part of Theorem 2.4 is true for an arbitrary dense and reflexive subsemigroup of $S$, then we get the following corollary.

Corollary 2.5 Let $B$ be a dense and reflexive subsemigroup of $S$. Then $B \omega \triangleleft S$.

Example 2.6 Let $S=\{a, b, c, e, f\}$ be the semigroup with the multiplication table given below:

\begin{tabular}{l|lllll}
\hline$\cdot$ & $e$ & $f$ & $a$ & $b$ & $c$ \\
\hline$e$ & $e$ & $e$ & $e$ & $b$ & $b$ \\
$f$ & $e$ & $f$ & $a$ & $b$ & $b$ \\
$a$ & $e$ & $a$ & $f$ & $b$ & $b$ \\
$b$ & $b$ & $b$ & $b$ & $e$ & $e$ \\
$c$ & $b$ & $c$ & $c$ & $e$ & $e$ \\
\hline
\end{tabular}

It is easy to see that $E$ is a dense and reflexive subsemigroup of $S$ but $E$ is not closed, since $e a \in E$ and $a \notin E$. Also, $N=\{a, e, f\}$ is normal. Indeed, the group congruence $\rho_{E}$ has two $\rho_{E}$-classes: $N$ and $\{b, c\}$, since $a e, e e, b b, b c \in E$ and $(e, b) \notin \rho_{E}$. Note also that $E \subset \operatorname{ker} \rho_{N}=N, E \neq N$ and $\rho_{E}=\rho_{N}$. It follows that there is no a one-toone correspondence between the set of all seminormal subsemigroups of $S$ and the set of all group congruences on $S$.

Remark 1 Obviously, every subgroup of a group is full and unitary but not every subgroup of a group is reflexive (for example: each two element subgroup of the group of all permutations of the six-element set $X$ is not reflexive). It is well known that a subgroup $H$ of a group $G$ is normal if and only if the relation $\rho_{H}$ is a congruence on $G$. We have a corresponding result:

Let $A$ be a closed subsemigroup of a semigroup $S$. Then $A$ is normal if and only if $\rho_{A} \in \mathcal{G C}(S)$.

Indeed, let $\rho_{A} \in \mathcal{G C}(S)$. From $A=A \omega$ and the second paragraph of the proof of Theorem 2.4 we obtain that $A=\operatorname{ker} \rho_{A}$. Thus $A \triangleleft S$ (Lemma 2.1). The converse of the result follows from Theorem 2.4.

The set of all group congruences on a semigroup $S$ (in general) does not form a lattice. Indeed, let $(\mathbb{R},+)$ be the semigroup of real positive numbers with respect to addition. Put $M=\mathbb{N}$ and $N=\{x, 2 x, 3 x, \ldots\}$, where $x \in \mathbb{R} \backslash \mathbb{Q}$. Then $M, N \triangleleft S$ but $M \cap N=\emptyset$.

We generalize now the results of Howie [12], LaTorre [16] and Hanumantha Rao and Lakshmi [9].

Theorem 2.7 Let $B$ be a seminormal subsemigroup of a semigroup $S, \rho \in \mathcal{C}(S)$. Then:

(i) $\rho \vee \rho_{B}=\rho_{B} \rho \rho_{B}$; 
(ii) $\rho \vee \rho_{B} \in \mathcal{G C}(S)$;

(iii) $(x, y) \in \rho \vee \rho_{B}$ if and only if $(a x, y b) \in \rho$ for some $a, b \in B$.

Proof (i). Since $\rho, \rho_{B} \subset \rho \vee \rho_{B}, \rho_{B} \rho \rho_{B} \subset \rho \vee \rho_{B}$. Also, $\rho_{B} \rho \rho_{B}$ is a reflexive, symmetric and compatible relation on $S$. We show that $\rho_{B} \rho_{B} \rho_{B}$ is transitive. Then $\rho \vee \rho_{B}=\rho_{B} \rho \rho_{B}$. Indeed, let $(r, s),(s, t) \in \rho_{B} \rho \rho_{B}$. Then (a) $(w, s),(s, x) \in \rho_{B}$; (b) $(y, w),(x, z) \in \rho$; (c) $(r, y),(z, t) \in \rho_{B}$ for some $w, x, y, z \in S$. From (a) we obtain $(w, x) \in \rho_{B}$, so $a w=x b$ for some $a, b \in B$. From (b) follows that $(a w, a y),(x b, z b) \in \rho$. Hence $(a y, z b) \in \rho$, since $a w=x b$. Finally, by $(c),(r, a y)$, $(z b, t) \in \rho_{B}$, since $B \subset \operatorname{ker} \rho_{B}$, so $(r, a y) \in \rho_{B},(a y, z b) \in \rho,(z b, t) \in \rho_{B}$. Thus $(r, t) \in \rho_{B} \rho \rho_{B}$, as required.

(ii). This is evident.

(iii). Let $(x, y) \in \rho \vee \rho_{B}$. Then $(x, r) \in \rho_{B},(r, s) \in \rho$ and $(s, y) \in \rho_{B}$ for some $r, s \in S$. Hence $a x=r b, c s=y d$ for some elements $a, b, c, d$ of $B$. Therefore $(c a) x=c(a x)=c(r b)=(c r b) \rho(c s b)=(c s) b=(y d) b=y(d b)$, where $c a, d b \in B$. Conversely, let $(a x, y b) \in \rho$ for some $a, b \in B$. Since $(x, a x),(y b, y) \in \rho_{B}$, then $(x, y) \in \rho_{B} \rho \rho_{B}=\rho \vee \rho_{B}$ (by (i)).

Let $A$ be a nonempty subset of a semigroup $S, \rho \in \mathcal{C}(S)$. Put

$$
A \rho=\{s \in S: \exists a \in A[(s, a) \in \rho]\} .
$$

Corollary 2.8 Let $B$ be a seminormal subsemigroup of a semigroup $S, \rho \in \mathcal{C}(S)$. Then $\operatorname{ker}\left(\rho \vee \rho_{B}\right)=(B \rho) \omega$. In particular, $(B \rho) \omega \triangleleft S$.

Proof Let $x \in \operatorname{ker}\left(\rho \vee \rho_{B}\right)$. Then there exists $b \in B$ such that $(x, b) \in \rho \vee \rho_{B}$, since $B \subset \operatorname{ker}\left(\rho \vee \rho_{B}\right)$. Hence $(a x, b c) \in \rho$ for some $a, c \in B$ (by Theorem 1.6(iii)). Thus $a x \in B \rho$. It follows that $x \in(B \rho) \omega$. Conversely, if $x \in(B \rho) \omega$, then $a x \in B \rho$ for some $a \in B \rho$, so $(a x, b),(a, c) \in \rho$ for some $b, c \in B$. It follows that $(c x, b) \in \rho$. Hence $((c c) x, c b) \in \rho$. Thus $(x, c) \in \rho \vee \rho_{B}$. Consequently, $x \in \operatorname{ker}\left(\rho \vee \rho_{B}\right)$.

Also, by Theorem 1.6(i) and Proposition 2.3(ii) in [15] we obtain the following (see Corollary 3.2 [15]) corollary.

Corollary 2.9 Every group congruence on a semigroup $S$ is dually right modular element of $\mathcal{C}(S)$.

Corollary 2.10 Let $B$ be a seminormal subsemigroup of a semigroup $S, \rho \in \mathcal{C}(S)$. Then $\rho \vee \rho_{B}=S \times S$ if and only if $(B \rho) \omega=S$.

Let $B$ be a seminormal subsemigroup of a semigroup $S, \rho_{1}, \rho_{2} \in \mathcal{C}(S)$. Suppose that $(x, y) \in\left(\rho_{1} \vee \rho_{B}\right) \cap\left(\rho_{2} \vee \rho_{B}\right)$. Then $(a x) \rho_{2}(y b)$, where $a, b \in B$. Moreover, $a x\left(\rho_{1} \vee \rho_{B}\right) x, x\left(\rho_{1} \vee \rho_{B}\right) y, y\left(\rho_{1} \vee \rho_{B}\right) y b$, so $a x\left(\rho_{1} \vee \rho_{B}\right) y b$. Thus $(c a x, y b d) \in \rho_{1}$, where $c, d \in B$. It follows that $($ caxd, cybd $) \in \rho_{1}$. Moreover, (caxd, cybd) $\in \rho_{2}$. Hence $(x d, c y) \in\left(\rho_{1} \cap \rho_{2}\right) \vee \rho_{B}$. Thus $(x, y) \in\left(\rho_{1} \cap \rho_{2}\right) \vee \rho_{B}$, since $\left(\rho_{1} \cap \rho_{2}\right) \vee \rho_{B}$ is a group congruence on $S$ and $c, d \in B \subset \operatorname{ker}\left(\left(\rho_{1} \cap \rho_{2}\right) \vee \rho_{B}\right)$. We have just shown 
that $\left(\rho_{1} \vee \rho_{B}\right) \cap\left(\rho_{2} \vee \rho_{B}\right) \subset\left(\rho_{1} \cap \rho_{2}\right) \vee \rho_{B}$. The converse inclusion is evident. Thus we may conclude that $\left(\rho_{1} \vee \rho_{B}\right) \cap\left(\rho_{2} \vee \rho_{B}\right)=\left(\rho_{1} \cap \rho_{2}\right) \vee \rho_{B}$.

We have the following theorem (see Theorem III.5.6 [21] and Theorem 4 [23]).

Theorem 2.11 Let $B$ be a seminormal subsemigroup of a semigroup $S$. Then the mapping $\phi: \mathcal{C}(S) \rightarrow \mathcal{G C}(S)$, where

$$
\rho \phi=\rho \vee \rho_{B}
$$

for every $\rho \in \mathcal{C}(S)$, is a (lattice) homomorphism of $\mathcal{C}(S)$ onto the (modular) lattice $\left[\rho_{B}, S \times S\right]$ of all group congruences on $S$ containing $\rho_{B}$.

Proof We have just proved that $\left(\rho_{1} \cap \rho_{2}\right) \phi=\rho_{1} \phi \cap \rho_{2} \phi$ for all $\rho_{1}, \rho_{2} \in \mathcal{C}(S)$. Clearly, $\left(\rho_{1} \vee \rho_{2}\right) \phi=\rho_{1} \phi \vee \rho_{2} \phi$ for all $\rho_{1}, \rho_{2} \in \mathcal{C}(S)$ and evidently $\phi$ is onto $\left[\rho_{B}, S \times S\right]$.

We have the following corollary (see Theorem 4.5 [15]).

Corollary 2.12 Let $B$ be a seminormal subsemigroup of a semigroup $S$. Then $\rho_{B}$ distributes over meet.

Let $S$ be a semigroup, $N \triangleleft S$. Put

$$
\mathcal{P}(S ; N)=\left\{A \subseteq S: A^{2} \subseteq A, N \subseteq A, A \omega=A\right\} .
$$

Also, denote $S / \rho_{N}$ by $S / N$. In particular, $\mathcal{P}(S / N ;\{N\})$ is the set of all subgroups of the group $S / N$. Remark that if $A \in \mathcal{P}(S ; N)$, then $A$ is full and dense.

The proofs of the following two propositions are standard and so we omit the proofs.

Proposition 2.13 Let $S$ be a semigroup, $N \triangleleft S$. Then there exists an inclusionpreserving bijection $\phi$ between the set $\mathcal{P}(S ; N)$ and the set $\mathcal{P}(S / N ;\{N\})$. Moreover, $M \in \mathcal{P}(S ; N)$ and $M \triangleleft S$ if and only if $M \phi \triangleleft S / N$.

Proposition 2.14 Let $\phi$ be an epimorphism of a semigroup $S$ onto a group $(G, \cdot, 1)$. Then:

(i) $\operatorname{Ker}(\phi)=\phi \phi^{-1}$ is a group congruence on $S$;

(ii) $N=\{1\} \phi^{-1} \triangleleft S$;

(iii) $\operatorname{Ker}(\phi)=\rho_{N}$.

Conversely, if $N \triangleleft S$, then $N$ is the kernel of the canonical homomorphism of $S$ onto $S / N$.

Example 2.15 We now describe all normal subsemigroups of the bicyclic semigroup $S=\mathbb{N}_{0} \times \mathbb{N}_{0}$, where $(k, l)(m, n)=(k-l+\max \{l, m\}, n-m+\max \{l, m\})$. It is known that every (non-identical) homomorphic image of the bicyclic semigroup is a cyclic group. Also, it is almost evident that $E_{S}=\{(0,0),(1,1),(2,2), \ldots\} \triangleleft S$ 
and $(k, l) \rho_{E}(m, n)$ if and only if $k+n=l+m$, so $S / \rho_{E} \cong(\mathbb{Z},+)$. It follows that $(i \mathbb{Z}) \phi^{-1}=\left\{(m, n) \in S:(m)_{i}=(n)_{i}\right\}$ for every $i \in \mathbb{N}$. The conclusion is that every cyclic group is a homomorphic image of the bicyclic semigroup.

We have also the following well known proposition (from group theory).

Proposition 2.16 Let $S$ be a semigroup; $M, N \triangleleft S$ and $M \subseteq N$. Then:

(i) $M \triangleleft N$;

(ii) $N / M \triangleleft S / M$;

(iii) $(S / M) /(N / M) \cong S / N$.

Every full and closed subsemigroup $A$ of an $E$-inversive semigroup $S$ is itself $E$-inversive. Indeed, let $a \in A$. Then $a x \in E_{S}=E_{A}$ for some $x \in S$, so $x \in A \omega=A$. Consequently, there is $x \in A$ such that $a x \in E_{A}$.

Finally, by way of contrast, we prove in the present section the following proposition which is valid for the class of all $E$-inversive semigroups.

Proposition 2.17 Let $S$ be an E-inversive semigroup, $N \triangleleft S$. Suppose also that a subsemigroup $M$ of $S$ is full and closed. Then:

(i) $M \cap N \triangleleft M$;

(ii) $N \triangleleft(M N) \omega$;

(iii) $M /(M \cap N) \cong(M N) \omega / N$.

Proof (i). It is clear that $E_{S} \subset M \cap N$, so $M \cap N$ is a full subsemigroup of $M$. Let $a, b \in M$ be such that $a b \in M \cap N$. Then $b a \in M$ and $b a \in N$ (since $N$ is reflexive in $S$ ). Hence $b a \in M \cap N$. Hence $M \cap N$ is reflexive in $M$. Further, if $x \in(M \cap N) \omega$, then $y x \in M \cap N$ for some $y \in M \cap N$, so $x \in M \cap N$ (because $N$ and $M$ are closed). Since $M \cap N$ is full and closed, then it is $E$-inversive, so it is dense in $M$. Thus $M \cap N \triangleleft M$.

(ii). We show that $(M N) \omega$ is a subsemigroup of $S$. Let $a, b \in(M N) \omega$. Then $m_{1} n_{1} a=m_{2} n_{2}$ for some $m_{1}, m_{2} \in M, n_{1}, n_{2} \in N$. Since $S$ is $E$-inversive, then $W\left(m_{1}\right) \neq \emptyset$. Hence $m m_{1}, m_{1} m \in E_{S} \subset M$ for some $m \in S$. Thus $m \in M$ (since $M$ is closed $),\left(m m_{1}\right) n_{1} a=\left(m m_{2}\right) n_{2}$. Therefore $\left(n_{1} a, m m_{2}\right) \in \rho_{N}$, since $m m_{1} \in E_{S} \subset N$, so $\left(a, m_{3}\right) \in \rho_{N}\left(m_{3} \in M\right)$. Similarly, $\left(b, m_{4}\right) \in \rho_{N}$ for some $m_{4} \in M$. It follows that $\left(a b, m_{5}\right) \in \rho_{N}$, where $m_{5} \in M$. Hence $n_{3} a b=m_{5} n_{4}$ for some $n_{3}, n_{4} \in N$. Thus $\left(m_{5} n_{3}\right) a b=\left(m_{5} m_{5}\right) n_{4}$. Consequently, $a b \in(M N) \omega$. Furthermore, $N \subset(M N) \omega$. Indeed, let $n \in N$. Then $n_{1} n=e n_{2}$ for some $e \in E_{S}, n_{1}, n_{2} \in N$. Hence we have $\left(e n_{1}\right) n=e n_{2} \in M N$, so $n \in(M N) \omega$. Consequently, $N \triangleleft(M N) \omega$ (since $\left.N \triangleleft S\right)$.

The proof of the condition (iii) is standard.

\section{Group congruences on an $E$-inversive semigroup}

Note that if a semigroup $S$ is $E$-inversive, then every full subsemigroup of $S$ is dense (since $E_{S}$ is dense), so a subsemigroup $A$ of $S$ is normal if and only if $A$ is full, 
reflexive and closed. It follows that $S$ has a least normal subsemigroup $U$. Thus the least group congruence on an arbitrary $E$-inversive semigroup exists. Denote it by $\sigma$ or $\sigma_{S}$. Then $\sigma=\rho_{U}$ and $\operatorname{ker} \sigma=U$ (Theorem 2.4).

Firstly, we have the following proposition.

Proposition 3.1 Let $S$ be an E-inversive semigroup. Then $\mathcal{G C}(S)=[\sigma, S \times S]$. Thus $\mathcal{G C}(S)$ is a complete sublattice of $\mathcal{C}(S)$.

Also, $\rho_{M} \vee \rho_{N}=\rho_{M} \rho_{N}=\rho_{N} \rho_{M}$ for all $M, N \triangleleft S$. Hence the lattice

$$
(\mathcal{G C}(S), \subseteq, \cap, \circ)
$$

is modular.

Proof The first part of the above proposition is clear. We show its second part. Let $a\left(\rho_{M} \rho_{N}\right) b$. Then $(a, c) \in \rho_{M},(c, b) \in \rho_{N}$, where $c \in S$. Take any $x \in W(c)$. Then $x c, c x \in E_{S},(c x a) \rho_{N}(b x a),(b x a) \rho_{M}(b x c)$, so $(a, b x a) \in \rho_{N},(b x a, b) \in \rho_{M}$. Hence $(a, b) \in \rho_{N} \rho_{M}$. Therefore $\rho_{M} \rho_{N} \subset \rho_{N} \rho_{M}$. We may equally well show the opposite inclusion. Consequently, $\rho_{M} \vee \rho_{N}=\rho_{M} \rho_{N}=\rho_{N} \rho_{M}$. In the light of Proposition I.8.5 [11], the lattice $(\mathcal{G C}(S), \subseteq, \cap, \circ)$ is modular.

Let $M, N$ be normal subsemigroups of a semigroup $S$. From Proposition 3.1 and Corollary 2.8 we obtain that $\operatorname{ker}\left(\rho_{M} \rho_{N}\right)=\operatorname{ker}\left(\rho_{N} \rho_{M}\right)=\left(M \rho_{N}\right) \omega=\left(N \rho_{M}\right) \omega$. In fact, if $S$ is $E$-inversive, then $\operatorname{ker}\left(\rho_{M} \rho_{N}\right)=\operatorname{ker}\left(\rho_{N} \rho_{M}\right)=M \rho_{N}=N \rho_{M}$. Indeed, let $x \in \operatorname{ker}\left(\rho_{M} \rho_{N}\right)$. Then $(x, e) \in \rho_{M} \rho_{N}$ for some $e \in E_{S}$. Hence $(x, n) \in \rho_{M}$, $(n, e) \in \rho_{N}$, where $n \in S$ (in fact, $\left.n \in \operatorname{ker} \rho_{N}=N\right)$. Thus $x \in N \rho_{M}$. Conversely, if $x \in N \rho_{M}$, then $(x, n) \in \rho_{M}$ for some $n \in N$. Hence $(x, n) \in \rho_{M},(n, e) \in \rho_{N}$, where $e \in E_{S}$. Thus $(x, e) \in \rho_{M} \rho_{N}$, that is, $x \in \operatorname{ker}\left(\rho_{M} \rho_{N}\right)$, so $\operatorname{ker}\left(\rho_{M} \rho_{N}\right)=N \rho_{M}$. Similarly, $\operatorname{ker}\left(\rho_{N} \rho_{M}\right)=M \rho_{N}$. This implies the required equalities. Also, $\operatorname{ker}\left(\rho_{M} \rho_{N}\right)=$ $(M N) \omega$. Indeed, let $x \in M \rho_{N}$. Then $n_{1} x=m n_{2}$ for some $n_{1}, n_{2} \in N, m \in M$. Hence $\left(m n_{1}\right) x \in M N$. Thus $x \in(M N) \omega$. We have proved that $\operatorname{ker}\left(\rho_{M} \rho_{N}\right) \subset(M N) \omega$. Conversely, let $x \in(M N) \omega$. Then $m_{1} n_{1} x=m_{2} n_{2}$ for some $m_{1}, m_{2} \in M, n_{1}, n_{2} \in N$. Since $S$ is $E$-inversive, then $m m_{1}=e \in E_{S} \subset M$ for some $m \in S$. It follows that $m \in M$ (since $M$ is closed), so $e n_{1} x=m m_{2} n_{2}$. Hence $\left(x, m m_{2}\right) \in \rho_{N}$. Thus $x \in M \rho_{N}=\operatorname{ker}\left(\rho_{M} \rho_{N}\right)$, so $(M N) \omega \subset \operatorname{ker}\left(\rho_{M} \rho_{N}\right)$, as exactly required.

In fact, we have just shown that in an arbitrary $E$-inversive semigroup $S$, $\rho_{(M N) \omega}=\rho_{M} \rho_{N}=\rho_{N} \rho_{M}=\rho_{(N M) \omega}$ for all $M, N \triangleleft S$. Moreover, notice that $\operatorname{ker}\left(\rho_{M} \cap \rho_{N}\right)=\operatorname{ker} \rho_{M} \cap \operatorname{ker} \rho_{N}=M \cap N(M, N \triangleleft S)$, so $\rho_{M} \cap \rho_{N}=\rho_{M \cap N}$ for $M, N \triangleleft S$. Consequently, the lattice $(\mathcal{N}(S), \subseteq, \cap, \vee)$, where $M \vee N=(M N) \omega$ for all $M, N \triangleleft S$, is isomorphic to the lattice $(\mathcal{G C}(S), \subseteq, \cap, \circ)$ (by the inclusion-preserving bijection $\phi$, see the proof of Theorem 2.4). Note also that the lattice $(\mathcal{N}(S), \subseteq, \cap, \vee)$ is complete (since it has the greatest element $S$ and the intersection of any nonempty family of normal subsemigroups of $S$ is a normal subsemigroup of $S$ ).

For terminology and elementary facts about lattices the reader is referred to the book [21] (Sect. I.2). The following result will be useful (see Exercise I.2.15(iii) in [21]).

Lemma 3.2 Every lattice isomorphism of complete lattices is a complete lattice isomorphism. 
From the above consideration we obtain the following theorem.

Theorem 3.3 Let $S$ be an E-inversive semigroup. Then there exists a (lattice) isomorphism $\phi$ between the lattice $(\mathcal{N}(S), \subseteq, \cap, \vee)$, where $M \vee N=(M N) \omega$ for all $M, N \triangleleft S$, and the lattice $(\mathcal{G C}(S), \subseteq, \cap, \circ)$. In fact, $\phi$ is defined by $N \phi=\rho_{N}$ for every $N \in \mathcal{N}(S)$. Moreover, $\phi$ is a complete lattice isomorphism.

Finally, we have the following proposition.

Proposition 3.4 Let $S$ be an E-inversive semigroup, $N \triangleleft S$. Then $(a, b) \in \rho_{N}$ if and only if $a b^{*} \in N$ for some (all) $b^{*} \in W(b)$.

Proof $(\Longrightarrow)$. Let $n a=b m$, where $n, m \in N$, and $b^{*} \in W(b)$. Then $n a b^{*}=b m b^{*}$. Since $b^{*} b m \in N$ and $N$ is reflexive, then $n a b^{*} \in N$. Hence $a b^{*} \in N \omega=N$.

$(\Longleftarrow)$. Let $a b^{*}=n \in N$ for some $b^{*} \in W(b)$. Then $a\left(b^{*} b\right)=n b$, so $(a, b) \in \rho_{N}$ (by Lemma 2.3).

\section{Group congruences on an $E$-semigroup}

First, we "generalize" some results from orthodox semigroups to $E$-semigroups (see Theorem VI.1.1 [11]).

Proposition 4.1 Let $S$ be a semigroup. The following conditions are equivalent:

(i) $S$ is an E-semigroup;

(ii) $\forall a, b \in S[W(b) W(a) \subseteq W(a b)]$.

Moreover, the condition (i) implies the following condition:

(iii) $\forall e \in E_{S}\left[W(e) \subseteq E_{S}\right]$.

If in addition $S$ is an $R$-semigroup, then the conditions (i)-(iii) are equivalent.

Proof The proof is closely similar to the proof of Theorem VI.1.1 [11].

Corollary 4.2 Let $S$ be an E-semigroup. Then:

(i) $\forall e \in E_{S}\left[W(e), V(e) \subseteq E_{S}\right]$;

(ii) $\forall a \in S, a^{*} \in W(a), e \in E_{S}\left[a e a^{*}, a^{*} e a \in E_{S}\right]$;

(iii) $\forall a \in S, a^{*} \in W(a), e, f \in E_{S}\left[e a^{*}, a^{*} e, e a^{*} f \in W(a)\right]$.

Proof (i). This follows from Proposition 4.1.

(ii). This follows from the proof of Proposition VI.1.4 [11].

(iii). Let $a \in S, a^{*} \in W(a), e, f \in E_{S}$. Since $e \in W(e)$ and $f \in W(f)$, then $e a^{*} \in W(e) W(a) \subseteq W(a e)$. Hence $e a^{*}=e a^{*} a e e a^{*}=\left(e a^{*}\right) a\left(e a^{*}\right)$. Therefore $e a^{*} \in W(a)$. Similarly, $a^{*} e \in W(a)$. Finally, $e a^{*} f \in W(e) W(a) W(f) \subseteq W($ fae $)$ and so $e a^{*} f=e a^{*} f f a e e a^{*} f=\left(e a^{*} f\right) a\left(e a^{*} f\right)$. Hence $e a^{*} f \in W(a)$. 
Proposition 4.3 Let $S$ be an E-inversive E-semigroup. Then

$$
\rho_{1, E}=\rho_{2, E}=\rho_{3, E}=\rho_{4, E}
$$

Proof Let $(a, b) \in \rho_{2, E}$ and $a^{*} \in W(a)$. Then $a e=f b$ for some $e, f \in E$. Moreover, $a^{*} f \in W(a)$ (Corollary 4.2(iii)), so $\left(a^{*} f\right) a, a\left(a^{*} f\right) \in E$. Further, $a^{*} f b=a^{*} a e \in E$. We have just shown that $x a, a x, x b \in E$ for some $x \in S$. Thus $\rho_{2, E} \subset \rho_{4, E}$.

On the other hand, if $x a, x b \in E$ for some $x \in S$, say $x a=e, x b=f$, then $(e f x) a(e f x)=e f(x a) e f x=e f x$, so $e f x \in W(a)$. Also, $f x b f x=f(x b) f x=f x$, i.e., $f x \in W(b)$. Hence ef $x \in W(b)$ (Corollary 4.2(iii)). Thus $W(a) \cap W(b) \neq \emptyset$. It follows that $a y, b y, y a, y b \in E$ for some $y \in S$. Dually, if $a x, b x \in E$ for some $x \in S$, then $a y, b y, y a, y b \in E$ for some $y \in S$. Thus $\rho_{4, E}=\rho_{1, E}$. In fact, we get $\rho_{4, E}=\rho_{1, E}=\{(a, b) \in S \times S: W(a) \cap W(b) \neq \emptyset\}$. Finally, if $x \in W(a) \cap W(b)$, then $a(x b)=(a x) b$ and $x b, a x \in E$. Thus $\rho_{2, E}=\rho_{4, E}=\rho_{1, E}$. We may equally well show that $\rho_{3, E}=\rho_{4, E}=\rho_{1, E}$. Consequently, $\rho_{1, E}=\rho_{2, E}=\rho_{3, E}=\rho_{4, E}$.

Lemma 4.4 Let $S$ be an E-inversive E-semigroup. Then:

(i) $\forall a \in S \exists e, f \in E_{S}[e a, a f \in \operatorname{Reg}(S)]$;

(ii) $\forall a \in S \exists r \in \operatorname{Reg}(S)[W(a) \cap W(r) \neq \emptyset]$.

Proof Let $a \in S, x \in W(a)$. Then $(a x) a, a(x a) \in \operatorname{Reg}(S)$, where $a x, x a \in E_{S}$, so (i) holds. Also, $r=\operatorname{axa} \in \operatorname{Reg}(S)$ and $x r x=x$. Thus $x \in W(a) \cap W(r)$.

Denote the above four relations from Proposition 4.3 by $\rho_{E}$. Recall that from the proof of Proposition 4.3 follows that $\rho_{E}=\{(a, b) \in S \times S: W(a) \cap W(b) \neq \emptyset\}$.

Theorem 4.5 In any E-inversive E-semigroup, $\sigma=\rho_{E}$. Moreover, $\operatorname{ker} \sigma=E_{S} \omega$. Thus $E_{S} \omega \triangleleft S$.

Proof It is clear that $\rho_{E}$ is an equivalence relation on $S$. Let $(a, b) \in \rho_{E}, c \in S$. Then $x \in W(a) \cap W(b)$. Take any $y \in W(c)$. In the light of Proposition 4.1,

$$
x y \in W(a) W(c) \cap W(b) W(c) \subseteq W(c a) \cap W(c b) .
$$

Hence $(c a, c b) \in \rho_{E}$. Thus $\rho_{E}$ is a left congruence on $S$. We may equally well show that $\rho_{E}$ is a right congruence on $S$. Also, if $e, f \in E_{S}$, then ee, ef $\in E_{S}$. Consequently, $(e, f) \in \rho_{E}$ for all $e, f \in E_{S}$. Lemma 4.4(ii) says that every $\rho_{E}$-class of $S$ contains a regular element. This implies that $S / \rho_{E}$ is a group.

Furthermore,

$$
x \in \operatorname{ker} \rho_{E} \Leftrightarrow \exists e \in E_{S}\left[(x, e) \in \rho_{E}\right] \Leftrightarrow \exists e, f, g \in E_{S}[f x=e g] \Leftrightarrow x \in E_{S} \omega,
$$

so $\operatorname{ker} \sigma=E_{S} \omega$. Thus $E_{S} \omega \triangleleft S$ (Theorem 2.4). Finally, $\rho_{E} \subseteq \rho_{N}$ for ever $N \triangleleft S$. Indeed, $E_{S} \subseteq N$. Hence $E_{S} \omega \subseteq N \omega=N$. Thus $\rho_{E}=\rho_{E_{S} \omega} \subseteq \rho_{N}$ (Theorem 2.4). Consequently, $\sigma=\rho_{E}$. 
Corollary 4.6 The least group congruence $\sigma$ on an E-inversive E-semigroup is given by

$$
\sigma=\left\{(a, b) \in S \times S: \exists e \in E_{S}[e a e=e b e]\right\} .
$$

Remark 2 Note that the condition " $\exists e \in E_{S}[e a e=e b e]$ " from the above corollary is equivalent to the apparently weaker condition " $\exists s \in S[s a s=s b s]$ ".

From Result 1.1 and Theorem 4.5 we obtain the following theorem.

Theorem 4.7 In any idempotent-surjective E-semigroup, $\sigma=\rho_{E}$.

Let $S$ be a semigroup. A congruence $\rho$ on $S$ is called idempotent pure if $e \rho \subseteq E_{S}$ for every $e \in E_{S}$. Note that if $S$ is idempotent-surjective, then $\rho$ is idempotent pure if and only if $\operatorname{ker} \rho=E_{S}$. Let $\mathcal{E}$ be an equivalence relation on $S$ induced by the partition: $\left\{E_{S}, S \backslash E_{S}\right\}$. Then $\mathcal{E}^{b}$ (defined in [13], see p. 27) is the greatest idempotent pure congruence on $S$. Put $\tau=\mathcal{E}^{b}$. Then (see [13], p. 28)

$$
\tau=\left\{(a, b) \in S \times S: \forall x, y \in S^{1}\left[x a y \in E_{S} \Longleftrightarrow x b y \in E_{S}\right]\right\} .
$$

Finally, if $S$ is $E$-inversive, then $\tau \subseteq \sigma$. Indeed, let $(a, b) \in \tau$ and $b^{*} \in W(b)$. Then $b b^{*} \in E_{S},\left(a b^{*}, b b^{*}\right) \in \tau$. Hence $a b^{*} \in E_{S} \subseteq \operatorname{ker} \sigma$. In the light of Proposition 3.4, $(a, b) \in \sigma$, as exactly required. In the following corollary we give an alternative proof of this fact.

Corollary 4.8 If $\rho$ is a congruence on an idempotent-surjective E-semigroup $S$, then $\operatorname{ker}(\rho \vee \sigma)=(\operatorname{ker} \rho) \omega$. In particular, $\tau \subseteq \sigma$.

Proof By Corollary 2.8, $\operatorname{ker}(\rho \vee \sigma)=\left(E_{S} \rho\right) \omega=(\operatorname{ker} \rho) \omega$. In particular,

$$
\operatorname{ker}(\tau \vee \sigma)=E_{S} \omega \subseteq \operatorname{ker} \sigma
$$

Hence $\tau \vee \sigma=\sigma$. Thus $\tau \subseteq \sigma$.

Let $\rho$ be a congruence on a semigroup $S$. By the trace $\operatorname{tr} \rho$ of $\rho$ we shall mean the restriction of $\rho$ to $E_{S}$. Also, we say that $\rho$ is idempotent-separating if $\operatorname{tr} \rho=1_{E_{S}}$. Edwards in [3] shows that if $S$ is an eventually regular semigroup, then the relation $\theta=\left\{\left(\rho_{1}, \rho_{2}\right) \in \mathcal{C}(S) \times \mathcal{C}(S): \operatorname{tr} \rho_{1}=\operatorname{tr} \rho_{2}\right\}$ is a complete congruence on $\mathcal{C}(S)$ and proves that every $\theta$-class $\rho \theta$ is a complete sublattice of $\mathcal{C}(S)$ with the maximum element

$$
\mu(\rho)=\left\{(a, b) \in S \times S:(a \rho, b \rho) \in \mu_{S / \rho}\right\}
$$

and the minimum element $1(\rho)$. Edwards generalizes some of these results for the class of all idempotent-surjective semigroups [4]. In fact, if $S$ is an arbitrary idempotent-surjective semigroup, then every $\theta$-class $\rho \theta$ is the interval $[1(\rho), \mu(\rho)]$, where $\mu$ is the maximum idempotent-separating congruence on $S$ (see [4] for more details). 
It is easily seen that the class of idempotent-surjective semigroups is closed under homomorphic images [10]. Using the obvious terminology we show next that every homomorphism of idempotent-surjective $E$-semigroups can be factored into a homomorphism preserving the maximal group homomorphic images and an idempotentseparating homomorphism. Firstly, we have need the following lemma.

Lemma 4.9 Let $\rho$ be a congruence on an idempotent-surjective E-semigroup $S$, $a, b \in S$. Then $(a \rho, b \rho) \in \sigma$ in $S / \rho$ implies $(a, b) \in \sigma$ if and only if $\rho \subseteq \sigma$.

Proof The proof is closely similar to the proof of Lemma III.5.9 [21].

Let $S$ be an idempotent-surjective $E$-semigroup, $\rho \in \mathcal{C}(S)$. Clearly, $(a, b) \in \sigma$ implies $(a \rho, b \rho) \in \sigma$. In the light of Lemma 4.9, if $\rho \subseteq \sigma$, then $(a, b) \in \sigma$ if and only if $(a \rho, b \rho) \in \sigma$. Hence $S / \sigma \cong(S / \rho) / \sigma$, that is, $S$ and $S / \rho$ have isomorphic maximal group homomorphic images. In that case, we may say that $\rho$ preserves the maximal group homomorphic images. Since for any congruence $\rho$ on $S$ we have $1(\rho) \subseteq \rho$, then we obtain the following factorization:

$$
S \rightarrow S / 1(\rho) \rightarrow S / \rho \cong(S / 1(\rho)) /(\rho / 1(\rho)) .
$$

The following proposition generalizes Proposition III.5.10 [21].

Proposition 4.10 Every homomorphism of idempotent-surjective E-semigroups can be factored into a homomorphism preserving the maximal group homomorphic images and an idempotent-separating homomorphism.

Proof Let $\rho$ be any congruence on an idempotent-surjective $E$-semigroup $S$. Since $\rho \subseteq S \times S$, then $1(\rho) \subseteq 1(S \times S)$. Clearly, $\sigma \in[1(S \times S), S \times S]$ and so $1(\rho) \subseteq \sigma$. It follows that the canonical epimorphism of $S$ onto $S / 1(\rho)$ preserves the maximal group homomorphic images. Finally, an epimorphism $\phi: S / 1(\rho) \rightarrow S / \rho$ (defined by the obvious way) is idempotent-separating, since $\operatorname{tr} \rho=\operatorname{tr}(1(\rho))$. The thesis of the proposition is a consequence of the above factorization.

\section{Group congruences on an $E$-unitary semigroup}

A nonempty subset $A$ of a semigroup $S$ is called left [right] unitary if $a s \in A$ [sa $\in A]$ implies $s \in A$ for every $a \in A, s \in S$. Also, we say that $A$ is unitary if it is both left and right unitary. Finally, a semigroup $S$ with $E_{S} \neq \emptyset$ is said to be $E$-unitary if $E_{S}$ is unitary.

Proposition 5.1 Let $S$ be a semigroup with $E_{S} \neq \emptyset$. The following conditions are equivalent:

(i) $S$ is E-unitary;

(ii) $E_{S}$ is left unitary;

(iii) $E_{S}$ is right unitary. 
Also, if $S$ is an E-unitary E-inversive semigroup, then $S$ is an E-semigroup.

Proof (i) $\Longrightarrow$ (ii). This is trivial.

(ii) $\Longrightarrow$ (iii). Let $s \in S, e \in E_{S}$. If $s e=f \in E_{S}$, then $f s e f=f$ and so we get $(e f s)(e f s)=e f s$, that is, efs $\in E_{S}$. Hence $f s \in E_{S}$. Thus $s \in E_{S}$.

(iii) $\Longrightarrow$ (i). We may equally well show like above that $E_{S}$ is left unitary. Thus the condition (i) holds.

Finally, let $S$ be an $E$-unitary $E$-inversive semigroup. If $e, f \in E_{S}, x \in W(e f)$, then $x$ ef $\in E_{S}$. Hence $x$ ef, $x \in E_{S}$. Thus ef $\in E_{S}$.

Corollary 5.2 Let $S$ be an E-inversive semigroup. Then the following conditions are equivalent:

(i) $S$ is E-unitary;

(ii) $\operatorname{ker} \sigma=E_{S}$;

(iii) $\tau=\sigma$.

In particular, if $S$ is an $E$-unitary $E$-inversive semigroup, then $E_{S} \triangleleft S$.

Proof (i) $\Longrightarrow$ (ii). In the light of Proposition 5.1 and Theorem 4.5, ker $\sigma=E_{S} \omega$. Also, $S$ is left unitary, that is, $E_{S}$ is closed. Thus $\operatorname{ker} \sigma=E_{S}$.

(ii) $\Longrightarrow$ (iii). We have mentioned above that $\tau \subseteq \sigma$. On the other hand, the main assumption implies that $\sigma$ is idempotent pure. Hence $\sigma \subseteq \tau$. Thus $\tau=\sigma$.

(iii) $\Longrightarrow$ (i). Let $a \in S, e, f \in E_{S}$. If $e a=f$, then $a \in \operatorname{ker} \sigma=\operatorname{ker} \tau=E_{S}$, that is, $E_{S}$ is left unitary. In the light of Proposition 5.1, $S$ is $E$-unitary.

Remark 3 Notice that if a semigroup is not $E$-inversive, then Corollary 5.2 is false. Indeed, let $F_{X}{ }^{1}$ be the free monoid on the set $X$. Then $F_{X}{ }^{1}$ is $E$-unitary but $\tau$ is induced by the partition $\left\{F_{X},\{1\}\right\}$. Thus $\tau$ is not a group congruence.

From Proposition 3.4 and Corollary 5.2 we obtain the following proposition.

Proposition 5.3 Let $S$ be an E-unitary E-inversive semigroup. Then $(a, b) \in \sigma$ if and only if $a b^{*} \in E_{S}$ for some (all) $b^{*} \in W(b)$.

Corollary 5.4 Let $A$ be an E-inversive subsemigroup of an $E$-unitary $E$-inversive semigroup $S$. Then $\sigma_{A}=\sigma_{S} \cap(A \times A)$.

Proof Clearly, $\sigma_{A} \subset \sigma_{S} \cap(A \times A)$. The converse follows from Proposition 5.3.

In [14] Howie and Lallement showed that $\sigma \cap \mathcal{H}=1_{S}$, when $S$ is an $E$-unitary regular semigroup. We prove a corresponding result.

Theorem 5.5 Let $S$ be an E-unitary E-inversive semigroup. Then $\sigma \cap \mathcal{H}=1_{S}$. Moreover, if in addition $E_{S}$ forms a semilattice, then $\sigma \cap \mathcal{L}=\sigma \cap \mathcal{R}=1_{S}$.

Proof Let $S$ be an $E$-unitary $E$-inversive semigroup. Suppose also that $E_{S}$ forms a semilattice. Then $E_{S}$ is normal (Corollary 5.2), so if $(a, b) \in \sigma \cap \mathcal{L}$, then $a x=e$, 
$b x=f \in E_{S}$ for some $x \in S$ (see Proposition 5.3) and $s a=b, t b=a$ for some $s, t \in S$. Hence $s e=s a x=b x=f \in E_{S}, t f=t b x=a x=e \in E_{S}$. Thus $s, t \in E_{S}$ (since $E_{S}$ is unitary), so since idempotents commute and $t a=t b$,

$$
a=t b=t(s a)=(t s) a=(s t) a=s(t a)=s(t b)=s a=b .
$$

We may equally well show that $\sigma \cap \mathcal{R}=1_{S}$.

If $S$ is $E$-unitary, then $E_{S}$ is normal, too. Let $(a, b) \in \sigma \cap \mathcal{H}$. By the above proof and its dual we conclude that $a=e b=b f$ and $b=g a=a h$ for some $e, f, g, h \in E_{S}$. In the light of Proposition 2 in [18], $a=b$.

Remark 4 The assumption that $S$ is an $E$-inversive semigroup is important. Indeed, let $S=\left(\mathbb{R}_{0},+\right)$ be the semigroup of nonnegative real numbers with respect to addition. Then $S$ is an $E$-unitary commutative semigroup. Put $M=\mathbb{N}_{0}$ and $N=\{0, x, 2 x, 3 x, \ldots\}$ (where $x \in \mathbb{R} \backslash \mathbb{Q}$ ). Then $M, N \triangleleft S$ but $M \cap N=\{0\}$ is not normal, so $S$ has no least group congruence.

The converse of Theorem 4.15 is not valid (in general). Indeed, let $S=\langle x\rangle$, where $x=(2345675)$ is a mapping of $\mathcal{T}(\{1,2, \ldots, 7\})$. Then $S=M(4,3)$ is the monogenic semigroup with index 4 and period 3 , say $S=\left\{x, x^{2}, \ldots, x^{6}\right\}$. Also, the cyclic subgroup $K_{x}$ of $S$ with the unit $e$ is equal $\left\{x^{4}, x^{5}, x^{6}=e\right\}$. Since $x^{3} e=x^{7} x^{2}=$ $x^{4} x^{2}=e$, then $S$ is not $E$-unitary. On the other hand, $\sigma$ is induced by the partition: $\left\{\left\{x, x^{4}\right\},\left\{x^{2}, x^{5}\right\},\left\{x^{3}, e\right\}\right\}$ and $\mathcal{H}$ by the partition: $\left\{K_{x},\{x\},\left\{x^{2}\right\},\left\{x^{3}\right\}\right\}$. Thus $\sigma \cap \mathcal{H}=1_{S}$.

From Theorem 5.5 and Corollary 5.2 we have the following corollary.

Corollary 5.6 Let $S$ be an E-unitary E-inversive semigroup. Then

$$
\sigma \cap \mathcal{H}=\tau \cap \mathcal{H}=1_{S}
$$

Moreover, if in addition $E_{S}$ forms a semilattice, then

$$
\sigma \cap \mathcal{L}=\tau \cap \mathcal{L}=\sigma \cap \mathcal{R}=\tau \cap \mathcal{R}=1_{S}
$$

Recall that a congruence $\rho$ on a semigroup $S$ is $E$-unitary if $S / \rho$ is $E$-unitary. In [5] the author described the least $E$-unitary congruence $\kappa$ on an idempotent-surjective semigroup. Also, for every congruence $\rho$ on an idempotent-surjective semigroup $S$ there exists the least $E$-unitary congruence $\kappa_{\rho}$ on $S$ containing $\rho$ [5].

Let $S$ be an idempotent-surjective semigroup, $N \triangleleft S$. Define the relation $\hat{\rho_{N}}$ on $\mathcal{C}(S)$ by the following rule: $\left(\rho_{1}, \rho_{2}\right) \in \hat{\rho_{N}} \Leftrightarrow \rho_{1} \vee \rho_{N}=\rho_{2} \vee \rho_{N}\left(\rho_{1}, \rho_{2} \in \mathcal{C}(S)\right)$. Then $\hat{\rho_{N}}$ is a congruence on $\mathcal{C}(S)$, since $\phi \phi^{-1}=\hat{\rho_{N}}$ (see Theorem 2.11).

Also, we prove the following proposition.

Proposition 5.7 Let $S$ be an idempotent-surjective semigroup, $N \triangleleft S, \rho \in \mathcal{C}(S)$. Then the elements $\rho, \kappa_{\rho}, \rho \vee \rho_{N}$ are $\hat{\rho_{N}}$-equivalent and $\rho \subseteq \kappa_{\rho} \subseteq \rho \vee \rho_{N}$. Moreover, the element $\rho \vee \rho_{N}$ is the largest in the $\hat{\rho_{N}}$-class $\hat{\rho_{0}}$. 
Proof Since $\kappa_{\rho}$ is the least $E$-unitary congruence containing $\rho$ and clearly $\rho \vee \rho_{N}$ is $E$-unitary, then $\rho \subseteq \kappa_{\rho} \subseteq \rho \vee \rho_{N}$. Hence $\rho \vee \rho_{N} \subseteq \kappa_{\rho} \vee \rho_{N} \subseteq \rho \vee \rho_{N}$. Therefore $\rho \vee \rho_{N}=\kappa_{\rho} \vee \rho_{N}$. Thus $\left(\rho, \kappa_{\rho}\right) \in \hat{\rho_{N}}$. Evidently, $\left(\rho, \rho \vee \rho_{N}\right) \in \hat{\rho_{N}}$. This implies the first part of the proposition. The second part is clear.

Remark 5 Recall from [22] that in the class of inverse semigroups not every $\hat{\sigma}$-class has a least element.

Finally, it is easy to see that the least $E$-unitary congruence $\kappa$ on an arbitrary $E$-inversive semigroup exists, too. We show that $\mathcal{H} \cap \sigma \subseteq \kappa$ in any $E$-inversive semigroup. Firstly, we have need the following useful proposition.

Proposition 5.8 Let $B$ be the least seminormal subsemigroup of an E-inversive semigroup $S$. If $\phi$ is an epimorphism of $S$ onto an $E$-unitary semigroup $T$, then $B \phi \subseteq E_{T}$.

Proof Put $A=\left(E_{T}\right) \phi^{-1}$. Clearly, $A$ is a full subsemigroup of $S$, so $A$ is dense. Further, if $x y \in A$, then $E_{T} \ni(x y) \phi=x \phi \cdot y \phi=y \phi \cdot x \phi=(y x) \phi$ (since $E_{T}$ is reflexive), so $y x \in A$. Hence $B \subseteq A$. Thus $B \phi \subseteq A \phi \subseteq\left(\left(E_{T}\right) \phi^{-1}\right) \phi \subseteq E_{T}$.

We may now prove the following equivalent theorem to Theorem 5.5.

Theorem 5.9 In any $E$-inversive semigroup $S, \mathcal{H} \cap \sigma \subseteq \kappa$. If in addition $E_{S}$ forms a semilattice, then $\mathcal{L} \cap \sigma \subseteq \kappa$ and $\mathcal{R} \cap \sigma \subseteq \kappa$.

Proof Indeed, $\sigma=\rho_{B}$, where $B$ is the least seminormal subsemigroup of $S$. Let $(a, b) \in \mathcal{H} \cap \sigma$. Then clearly $(a \kappa, b \kappa) \in \mathcal{H}^{S / \kappa}$. Also, $a x=y b$ for some $a, b \in B$. In the light of Proposition 5.8, $(a \kappa)(x \kappa)=(y \kappa)(b \kappa)$, where $a \kappa, b \kappa \in E_{S / \kappa}$. Hence $(a \kappa, b \kappa) \in \mathcal{H}^{S / \kappa} \cap \sigma_{S / \kappa}=1_{S / \kappa}$ (Theorem 5.5). Thus $\mathcal{H} \cap \sigma \subseteq \kappa$, as required.

\section{Group congruences on an eventually regular semigroup}

Group congruences on eventually regular semigroups were described in [9] by Hanumantha Rao and Lakshmi. In the paper [9] the following definition was introduced: a subset $A$ of $S$ is called self-conjugate if $x^{r(x)-1}\left(x^{r(x)}\right)^{*} A x \subseteq A$ and $x A x^{r(x)-1}\left(x^{r(x)}\right)^{*} \subseteq A$ for all $x \in S,\left(x^{r(x)}\right)^{*} \in V\left(x^{r(x)}\right)$. We say that $A$ is selfconjugate if the former condition holds.

Lemma 6.1 Let $N$ be a subsemigroup of an eventually regular semigroup $S$. Then $N$ is normal if and only if $N$ is full, self-conjugate and closed.

Proof Let $N$ be normal, $x \in S,\left(x^{r(x)}\right)^{*} \in V\left(x^{r(x)}\right)$. Then $N$ is full and closed. Also, $x^{r(x)}\left(x^{r(x)}\right)^{*} N \subseteq E N \subseteq N$, so $x^{r(x)-1}\left(x^{r(x)}\right)^{*} N x \subseteq N$, since $N$ is reflexive.

Let $N$ be full, self-conjugate and closed, $x y \in N,\left(x^{r(x)}\right)^{*} \in V\left(x^{r(x)}\right)$. Then $x^{r(x)-1}\left(x^{r(x)}\right)^{*}(x y) x \in x^{r(x)-1}\left(x^{r(x)}\right)^{*} N x \subseteq N$, i.e., $\left(x^{r(x)-1}\left(x^{r(x)}\right)^{*} x\right)(y x) \in N$, where $x^{r(x)-1}\left(x^{r(x)}\right)^{*} x \in E_{S} \subseteq N$. Hence $y x \in N \omega=N$, so $N$ is reflexive. Thus $N \triangleleft S$. 
Lemma 6.2 Let $S$ be an eventually regular semigroup, $N \triangleleft S$. Then

$$
\rho_{N}=\left\{(a, b) \in S \times S: \exists\left(b^{r(b)}\right)^{*} \in V\left(b^{r(b)}\right)\left[a b^{r(b)-1}\left(b^{r(b)}\right)^{*} \in N\right]\right\} .
$$

Proof Let $(a, b) \in \rho_{N}$ and $\left(b^{r(b)}\right)^{*} \in V\left(b^{r(b)}\right)$. Then $n a=b m$ for some $n, m \in N$. Hence $n a b^{r(b)-1}\left(b^{r(b)}\right)^{*}=b m b^{r(b)-1}\left(b^{r(b)}\right)^{*}$. Also, since $b^{r(b)-1}\left(b^{r(b)}\right)^{*} b \in E_{S}$, then $m b^{r(b)-1}\left(b^{r(b)}\right)^{*} b \in N E_{S} \subseteq N$, so $n a b^{r(b)-1}\left(b^{r(b)}\right)^{*}=b m b^{r(b)-1}\left(b^{r(b)}\right)^{*} \in N$, since $N$ is reflexive. Consequently, $a b^{r(b)-1}\left(b^{r(b)}\right)^{*} \in N \omega=N$.

Conversely, let $a, b \in S,\left(b^{r(b)}\right)^{*} \in V\left(b^{r(b)}\right)$ and $a b^{r(b)-1}\left(b^{r(b)}\right)^{*}=n \in N$. Then $a\left(b^{r(b)-1}\left(b^{r(b)}\right)^{*} b\right)=n b$, where $b^{r(b)-1}\left(b^{r(b)}\right)^{*} b \in E_{S} \subseteq N$. Hence $(a, b) \in \rho_{N}$.

We have the following corollary (see Theorem 1 [9]).

Corollary 6.3 Let $S$ be an eventually regular semigroup, $N \triangleleft S$. Then

$$
\rho_{N}=\left\{(a, b) \in S \times S: \exists\left(b^{r(b)}\right)^{*} \in V\left(b^{r(b)}\right)\left[a b^{r(b)-1}\left(b^{r(b)}\right)^{*} \in N\right]\right\}
$$

is a group congruence on $S$.

Finally, we give some remarks concerning group congruences on inverse semigroups. Firstly, consider the following result (see Exercise 7(ii) [11], p. 181).

Statement 6.4 An inverse subsemigroup $N$ of an inverse semigroup $S$ is normal if and only if $(N x) \omega=(x N) \omega$ for every $x \in S$.

This result is false. Indeed, let $S$ be a Clifford semigroup. Put $N=\mathcal{Z}(S)$, where $\mathcal{Z}(S)=\{s \in S: \forall a \in S[s a=a s]\}$. Clearly, $N$ is a full subsemigroup of $S$. Also, $N$ is self-conjugate. If the result is valid, then $N$ is normal (since $N x=x N$ for every $x \in S$ ). Hence $\rho_{N}=S \times S=\rho_{S}$, when $S=S^{0}$. It follows that every Clifford semigroup is commutative, a contradiction. Consequently, we conclude that the above result is false. Moreover, the assumptions of the result and the conditions: " $N$ is full" and " $N$ is self-conjugate" do not imply that $(N x) \omega=(x N) \omega$ for every $x \in S$.

It is clear that every subgroup of a group is full and closed. We prove now a correct version of the above statement.

Proposition 6.5 A full and closed inverse subsemigroup $N$ of an inverse semigroup $S$ is normal if and only if $(N x) \omega=(x N) \omega$ for every $x \in S$.

Proof It is easy to see that if $N$ is normal, then $(N x) \omega=(x N) \omega$ for every $x \in S$.

Conversely, let $(N x) \omega=(x N) \omega$ for every $x \in S$. It is easy to check that two relations $\rho_{1}=\left\{(a, b) \in S \times S: a b^{-1} \in N\right\}$ and $\rho_{2}=\left\{(a, b) \in S \times S: a^{-1} b \in N\right\}$ are equivalences on $S$ and that $x \rho_{1}=(N x) \omega, x \rho_{2}=(x N) \omega$ for every $x \in S$. Also, $\rho_{1}$ is right compatible and $\rho_{2}$ is left compatible. Indeed, we show first that the equality $(A(B \omega)) \omega=(A B) \omega$ holds for all $A, B \subseteq S$. Recall from [11] that

$$
H \omega=\{s \in S: \exists h \in H[h \leq s]\} \quad(H \subseteq S),
$$


where $\leq$ is the so-called natural partial order on (an inverse semigroup) $S$ (i.e., $\left.a \leq b \Longleftrightarrow \exists e \in E_{S}[a=e b]\right)$. Notice that $\leq$ is compatible. Let $x \in(A(B \omega)) \omega$. Then $a y \leq x$ for some $a \in A, y \in B \omega$ (that is, $b \leq y$ for some $b \in B$ ). Hence $a b \leq a y \leq x$. Thus $x \in(A B) \omega$. We have just proved that $(A(B \omega)) \omega \subset(A B) \omega$. The opposite inclusion is clear. Let now $(a, b) \in \rho_{2}, c \in S$. Then $(a N) \omega=(b N) \omega$ and so $(c(a N) \omega) \omega=(c(b N) \omega) \omega$. Therefore $(c a N) \omega=(c b N) \omega$. Thus $\rho_{2}$ is a left congruence on $S$. We may equally well show that $\rho_{1}$ is a right congruence on $S$. Since $(N x) \omega=(x N) \omega$ and $x \rho_{1}=(N x) \omega, x \rho_{2}=(x N) \omega$ for every $x \in S$, then $\rho_{1}=\rho_{2}$ is a congruence on $S$. Put for simplicity $\rho=\rho_{1}=\rho_{2}$. Finally, if $e \in E_{S}$, then $E_{S} \subseteq N=N \omega=(e N) \omega$. Hence $\rho$ is a group congruence on $S$ and $\operatorname{ker} \rho=N$. Thus $N \triangleleft S$, as required.

Corollary 6.6 A Clifford semigroup $S$ is commutative if and only if $\mathcal{Z}(S)$ is closed in $S$ (i.e., if and only if for every $s \in S$ there exists $z \in \mathcal{Z}(S)$ such that $z \leq s$ ).

Lemma 6.7 Let $S$ be a finite inverse semigroup with semilattice of idempotents $E$. Then $E \omega=S$ if and only if $S$ has zero.

Proof It is clear that if $S$ has zero, then $E \omega=S$. Conversely, let $E \omega=S$. Since $E$ is finite, then $E$ has the least idempotent with respect to the natural partial order, say 0 . Let $s \in S=E \omega$. Then $e=f s$ and $e=s g$ for some $e, f, g \in E$ (see Proposition V.2.2 in [11]). Hence $0=0 s=s 0$. Thus $S=S^{0}$, as required.

By an analogy to groups we may introduce the concept of a $\sigma$-simple inverse semigroup in the class of finite inverse semigroups without 0. From Lemma 6.7 follows that every finite inverse semigroup $S$ without zero has at least one non-universal group congruence, so $S$ has exactly one non-universal group congruence if and only if $S / E \omega$ is a simple group. Hence we may say that a finite inverse semigroup $S$ without zero is $\sigma$-simple if $S / E \omega$ is a simple group. This definition is equivalent to the following definition: $S$ is $\sigma$-simple if $S$ has exactly two normal subsemigroups, namely: $E \omega$ and $S$.

Example 6.8 Let $(E, \leq)$ be a chain with the least element 0 . Put $S=E \cup\{a\}$, where $a \notin E$ and $a a a=a$. Assume also that $a a=0$. Hence $a=a a a=0 a=a 0$. It is easy to see that if a binary operation on $S$ is associative, then $e a=a e=a$ for every $e \in E_{S}$. For example, $e a=e(0 a)=(e 0) a=0 a=a$. Conversely, it is straightforward to verify that such defined binary operation is associative. Thus $S$ is a semigroup. Since $a=a^{-1}$, then $S$ is an inverse semigroup. Finally, $E=E \omega$, so $S / E=\{E,\{a\}\}$.

\section{The hypercore of a semigroup}

In [8] Hall and Munn studied the hypercore of a semigroup. In this section we give some remarks on the hypercore of $E$-inversive $E$-semigroups and inverse semigroups.

Let $S$ be a semigroup with $E_{S} \neq \emptyset$. Denote by $\wp_{S}$ the set of all subsemigroups $A$ of $S$ such that $A$ has no cancellative congruences except the universal congruence. 
Note that $\{e\} \in \wp_{S}$ for every $e \in E_{S}$. Define the hypercore hyp $(S)$ of $S$, as follows: $\operatorname{hyp}(S)=\left\langle\bigcup\left\{A: A \in \wp_{S}\right\}\right\rangle$ [8]. Furthermore, by the core core $(S)$ of an $E$-inversive semigroup $S$ we shall mean $\operatorname{ker} \sigma$.

In [8] the authors showed the following two results.

Result 7.1 Let $S$ be an E-inversive semigroup. Then:

(i) $\operatorname{hyp}(S) \in \wp_{S}$;

(ii) $\operatorname{hyp}(S)$ is full and unitary;

(iii) $\forall \rho \in \mathcal{G C}(S)[\operatorname{hyp}(S) \subseteq \operatorname{ker} \rho]$.

Result 7.2 In any E-inversive semigroup $S$, hyp $(S)$ is the greatest E-inversive subsemigroup of $S$ with no non-universal group congruence.

Let $U$ be the least full unitary subsemigroup of an $E$-inversive semigroup $S$. Clearly, $U \subseteq \operatorname{hyp}(S) \subseteq \operatorname{core}(S)$.

Finally, we have the following proposition.

Proposition 7.3 Let $S$ be an E-inversive E-semigroup such that $1_{S} \notin \mathcal{G C}(S)$. Then $U=\operatorname{hyp}(S)=\operatorname{core}(S)=E_{S} \omega$. In particular, $E_{S} \omega$ has no non-universal group congruence.

If in addition $S$ is an inverse semigroup and $E_{S} \omega$ is finite, then $E_{S} \omega$ is an inverse semigroup with zero. In particular, every finite inverse semigroup $S$ (which is not a group) contains exactly one normal inverse subsemigroup with zero.

Proof Let $S$ be an $E$-inversive $E$-semigroup. Then core $(S)=E_{S} \omega$ (Theorem 4.5). Since $E_{S} \subseteq U$ and $U$ is closed, then $E_{S} \omega \subseteq U$, so $U=\operatorname{hyp}(S)=\operatorname{core}(S)=E_{S} \omega$. In the light of Result 7.2, $E_{S} \omega$ has no non-universal group congruence.

If $S$ is an inverse semigroup, then obviously $U=\operatorname{hyp}(S)=\operatorname{core}(S)=E_{S} \omega$ has no non-universal group congruence. Finally, if $E_{S} \omega$ is finite, then $E_{S} \omega$ has zero (Lemma 6.7). The rest of the proposition is now immediate.

Open Access This article is distributed under the terms of the Creative Commons Attribution License which permits any use, distribution, and reproduction in any medium, provided the original author(s) and the source are credited.

\section{References}

1. Clifford, A.H., Preston, G.B.: The Algebraic Theory of Semigroups, Vol. II. Math. Surveys, vol. 7. Am. Math. Soc., Providence (1967)

2. Edwards, P.M.: Eventually regular semigroups. Bull. Aust. Math. Soc. 28, 23-38 (1983)

3. Edwards, P.M.: On the lattice of congruences on an eventually regular semigroup. J. Aust. Math. Soc. 38A, 281-286 (1985)

4. Edwards, P.M.: Maximizing a congruence with respect to its partition of idempotents. Semigroup Forum 39, 257-262 (1989)

5. Gigoń, R.S.: On the lattice of congruences on idempotent-regular-surjective semigroups. Accepted for publication in Commun. Algebra

6. Gomes, G.: A characterization of the group congruences on a semigroup. Semigroup Forum 46, 48-53 (1993) 
7. Hall, T.E.: On regular semigroups. J. Algebra 24, 1-24 (1973)

8. Hall, T.E., Munn, W.D.: The hypercore of a semigroup. Proc. Edinb. Math. Soc. 28, 107-112 (1985)

9. Hanumantha Rao, S., Lakshmi, P.: Group congruences on eventually regular semigroups. J. Aust. Math. Soc. 45A, 320-325 (1988)

10. Higgins, P.M.: The converse of Lallement's lemma. In: Semigroups and Applications, St. Andrews, 1997, pp. 78-86. World Scientific, River Edge (1998)

11. Howie, J.M.: An Introduction to Semigroup Theory. Academic Press, London (1984)

12. Howie, J.M.: The maximum idempotent-separating congruence on an inverse semigroup. Proc. Edinb. Math. Soc. 14, 71-79 (1964)

13. Howie, J.M.: Fundamentals of Semigroup Theory. Oxford University Press, New York (1995)

14. Howie, J.M., Lallement, G.: Certain fundamental congruences on a regular semigroup. Proc. Glasgow Math. Soc. 7, 145-159 (1966)

15. Jones, P.R.: Joins and meets of congruences on a regular semigroup. Semigroup Forum 30, 1-16 (1984)

16. LaTorre, D.R.: Groups congruence on regular semigroups. Semigroup Forum 24, 327-340 (1982)

17. Meakin, J.: Congruences on orthodox semigroups II. J. Aust. Math. Soc. 13, 259-266 (1972)

18. Mitsch, H.: A natural partial order for semigroups. Proc. Am. Math. Soc. 97, 384-388 (1986)

19. Mitsch, H., Petrich, M.: Basic properties on E-inversive semigroups. Commun. Algebra 28, 5169$5182(2000)$

20. Mitsch, H., Petrich, M.: Restricting idempotents in E-inversive semigroups. Acta Sci. Math. (Szeged) 67, 555-570 (2001)

21. Petrich, M.: Inverse Semigroups. Wiley, New York (1984)

22. Reilly, N.R.: Update on the problems in "Inverse semigroups" by M. Petrich. Lect. Notes Math. 1320, 287-299 (1986)

23. Yang, Y., Tian, Z.: The group congruences on E-inversive semigroups. Int. J. Contemp. Math. Sci. 6(1-4), 103-108 (2011) 